Psychiatria clin. 1977;10:1-3

\title{
Contents, Vol. 10, No. 1-3, 1977
}

\section{Index}

Vorwort 4

Foreword 5

Reiter, L. und Strotzka, H.: Der Begriff der Krise. Ideengeschichtliche Wur-zeln und aktuelle Probleme des Krisenbegriffes 7

Häfner, H.: Psychiatrische Krisenintervention - Umsetzung in psychia-trischen Einrichtungen.

Bericht über Entwicklungstendenzen in denwesteuropäischen Ländern und den USA

Temkov, I.: Psychiatrische Krisenintervention. Organisatorische und administrative

Bedingungen im staatlichen Gesundheitsdienst 64

Sonneck, G. undRingel, E.: Technik der Krisenintervention 85

Ciompi, L.: Gedanken zu den therapeutischen Möglichkeiten einer Technikder provozierten Krise 96

Bellak, L.: Kombinierte Psycho- und Pharmakotherapie unter besondererBerücksichtigung von Kurz- und Notfalltherapie 102

Friedrich, M.H.: Krisenintervention bei Jugendlichen. Ein soziales Modell in

Wien 114

Berger, E.: Teamarbeit in der Jugendfürsorge als Instrument der Krisenintervention

Sonneck, G.: Suizidhandlungen und Kriseninterventionen. Zusammen-fassung von Ergebnissen des 7. Donausymposions für Psychiatrie .... 131

G. Gabriel

E.; Reiter

L. und Springer

A.: Krisenintervention in der Psychiatrie. Zusammenfassung der Vorträge und Diskussionen 140

Gabriel

E.; Reiter

L.

and Springer

A.: Crisis Intervention in Psychiatry.Summary of the Lectures and Discussions 